\title{
Solid-phase extraction and high-performance liquid chromatographic determination of polyphenols in apple musts and ciders
}

\author{
B. Suárez*, A. Picinelli, J.J. Mangas \\ Instituto de Experimentación y Promoción Agraria, 33300, Villaviciosa Asturias, Spain
}

Received 2 August 1995; revised 13 October 1995; accepted 13 October 1995

\begin{abstract}
An improved analytical method was developed for the determination of polyphenols in the apple products must and cider. Phenolic compounds were fractionated into neutral and acidic groups by means of a solid-phase extraction method. The analytical method proposed was effective for the quantitation of phenolic compounds; recoveries between $84 \%$ and $111 \%$ were obtained, and the relative standard deviation was usually less than $5 \%$.
\end{abstract}

Keywords: Solid-phase extraction; Apple products; Food analysis; Polyphenols; Flavonoids; Phenolic acids

\section{Introduction}

Low-molecular-mass apple polyphenols have been exhaustively studied due to their contribution to the color and flavor of apple juices; particularly $o$ diphenols, catechins and their polymers, and chlorogenic acid. The contribution of neutral phenolics to the astringency and bitterness properties of beverages has also been described [1].

The isolation and quantitation of polyphenols in apple and processed apple products is difficult due to their chemical complexity. Different methods have been used to fractionate them prior to chromatographic analysis. Salagoity-Auguste and Bertrand [2] separated neutral and acidic phenolic compounds after extraction with ethylacetate at two different $\mathrm{pH}$ values. This method was further applied to apple

\footnotetext{
${ }^{*}$ Corresponding author.
}

juices analysis [3,4]. However, recoveries for procyanidins and flavonols were poor. Sephadex LH20 was extensively used with good results to separate procyanidins from phenolic acids in apples $[5,6]$ and ciders [7]. However, this technique is usually timeconsuming, and low and variable recovery of (-)epicatechin was reported [8]. Solid-phase extraction with $\mathrm{C}_{18}$ cartridges makes the sample preparation faster and easier. It was employed to determine, with good recovery, 4-vinyl guaiacol and ferulic acid in orange juice [9], and phenolic acids and flavonoids in grapes [10] and apples [11,12]. Also, Oszmianski et al. [13] developed a single method for the fractionation of phenolic acids, flavonols, anthocyanin monomers and polymers, procyanidins and catechins in red wine, using a $\mathrm{C}_{18}$ Sep-Pak cartridge.

RP-HPLC with $\mathrm{C}_{18}$ columns, is the most popular technique for the analysis of polyphenols in different foods. However, the separation of procyanidins is not satisfactory with these phases. Lea [14] resolved

$0021-9673 / 96 / \$ 15.00$ (C) 1996 Elsevier Science B.V. All rights reserved SSDI $0021-9673(95) 01175-7$ 
these compounds efficiently using a $\mathrm{C}_{6}$ reversedphase.

In this paper, a solid-phase extraction and HPLC method for quantitative determination of flavonoids and phenolic acids in apple musts and ciders is described.

\section{Experimental}

\subsection{Reagents}

Standards of (+)-catechin, (-)-epicatechin, phloridzin and phenolic acids were purchased from Sigma Chemical (St. Louis, MO, USA), and glycosides of quercetin were from Extrasyntèse (Genay, France); phloretin xyloglucoside and procyanidins were kindly furnished by Dr. A. Lea (Reading, UK). Solvents employed were from Panreac, HPLC quality (Barcelona, Spain), and degassed with helium prior to use.

\subsection{Preparation of the samples}

Apple must was obtained by means of a domestic juice-maker. The juice was centrifuged at $12000 \mathrm{~g}$ for $5 \mathrm{~min}$ and frozen at $-20^{\circ} \mathrm{C}$ until its analytical determination. Cider samples were dealcoholized under vacuum at $35^{\circ} \mathrm{C}$, previous to the fractionation process.

\subsection{Total polyphenol analysis}

Analyses were done by the Folin-Ciocalteu method [15].

\subsection{Extraction and fractionation of phenolic compounds}

\subsubsection{Preconditioning of solid-phase supports}

Sep-Pak classic (360 mg; Waters, Milford, MA, USA) and Sep-Cartridge ( $300 \mathrm{mg}$; Lida, Kenosha, WI, USA), were preconditioned for neutral phenolics by sequentially passing $8 \mathrm{ml}$ of methanol and $4 \mathrm{ml}$ of water adjusted to $\mathrm{pH}$ 7.0. For acidic phenolics, cartridges were preconditioned by passing $4 \mathrm{ml}$ of $0.01 \mathrm{M} \mathrm{HCl}$ instead of water. Extra-Sep $\mathrm{C}_{18}$ columns (1000 mg; Lida, Kenosha, WI, USA) were pre- conditioned for neutral phenols by sequentially passing $25 \mathrm{ml}$ of methanol and $10 \mathrm{ml}$ of water adjusted to $\mathrm{pH}$ 7.0. Solid-phase supports for phenolic acids were preconditioned by sequentially passing $25 \mathrm{ml}$ of methanol and $10 \mathrm{ml}$ of $0.01 \mathrm{M} \mathrm{HCl}$.

\subsubsection{Fractionation}

The optimized fractionation method was developed for Extra-Sep $\mathrm{C}_{18}$ columns and was as follows. A suitable volume of sample (to load $8 \mathrm{mg}$ of total polyphenols per gram of solid phase), containing $10 \mathrm{~g} / \mathrm{l}$ ascorbic acid to avoid oxidation of polyphenols, was adjusted to $\mathrm{pH} 7.0$ with $\mathrm{NaOH}$, loaded onto the $\mathrm{C}_{18}$ neutral fractionating column and washed with $10 \mathrm{ml}$ of $\mathrm{pH} 7.0$ water. The effluent portion was adjusted to $\mathrm{pH} 2.0$ with $2 \mathrm{M} \mathrm{HCl}$, passed through the preconditioned acidic column, and washed with $5 \mathrm{ml}$ of $0.01 \mathrm{M} \mathrm{HCl}$, in the case of musts. The adsorbed fractions were eluted with 12 $\mathrm{ml}$ of methanol, and evaporated till dryness at $35^{\circ} \mathrm{C}$. The residue was redissolved in $0.5 \mathrm{ml}$ of methanol, filtered through a $0.22-\mu \mathrm{m}$ polyvinylidene difluorure filter (PVDF, Lida, Kenosha, WI, USA), and $10 \mu \mathrm{l}$ were injected onto the HPLC system.

\subsection{HPLC analysis}

Analyses were performed with a Waters system, equipped with a 712 automatic injector, M510 pumps, a Millenium v.2.0 software data module and a 996 diode-array detector.

Separation of polyphenols (neutral and acidic) in musts and neutral phenols in ciders, was carried out on a reversed-phase Spherisorb Hexyl cartridge $(250 \times 4.6 \mathrm{~mm}$ I.D., $5 \mu \mathrm{m}$ particle size; Tracer Analitica, Teknokroma, Barcelona, Spain) at $30^{\circ} \mathrm{C}$, using as solvents $2 \mathrm{~m} M$ phosphoric acid (solvent $\mathrm{A}$ ) and methanol (solvent B), using the linear gradient elution of Table 1.

Acidic compounds in ciders were analysed using a Nucleosil $120 \mathrm{C}_{18}$ column $(250 \times 4.6 \mathrm{~mm}$ I.D., $3 \mu \mathrm{m}$ particle size; Tracer Analitica, Teknokroma) at $25^{\circ} \mathrm{C}$, using 2\% acetic acid (solvent $\mathrm{A}$ ) and methanol (solvent B) as mobile phases. The gradient used was linear: $0-45 \% \mathrm{~B}$ in $55 \mathrm{~min}$, at a flow-rate of 0.8 $\mathrm{ml} / \mathrm{min}$.

Identification of each compound was performed by its retention time and by spiking with the standards, 
Table 1

Gradient elution for the separation of polyphenols (neutral and acidic) in apple musts and neutral phenols in ciders

\begin{tabular}{clc}
\hline$t(\min )$ & Flow-rate $(\mathrm{ml} / \mathrm{min})$ & $\%$ B \\
\hline 0 & 1.20 & 2 \\
15 & 1.20 & 11 \\
20 & 1.20 & 18.5 \\
23 & 1.20 & 20.3 \\
25 & 0.80 & 21.5 \\
55 & 0.80 & 39.5 \\
\hline
\end{tabular}

Solvent A was $2 \mathrm{mM}$ phosphoric acid; solvent B was methanol.

under the same conditions. Additionally, the peak purity was checked by means of the PDA Millenium software spectral contrast facilities. Quantitation was carried out at different wavelengths: $320 \mathrm{~nm}$ for cinnamic acids, $360 \mathrm{~nm}$ for flavonols, and $280 \mathrm{~nm}$ for the rest of polyphenols.

\section{Results and discussion}

\subsection{Gradient optimization for neutral phenolics in musts and ciders}

Previous tests on two $\mathrm{C}_{18}$ stationary phases [4] showed a partial overlapping between procyanidins $\mathrm{C} 1$ and a procyanidin tetramer. Lea $[14,16]$ obtained a good resolution of cider and apple procyanidins using a reversed-phase $C_{6}$ column.

The gradient elution profile described elsewhere [4] was adapted to the Hexyl cartridge and optimized, with the aim to achieve a value of $R_{\mathrm{s}} \geq 1$. Isocratic studies of flow-rate between 1.0 and 1.5 $\mathrm{ml} / \mathrm{min}$ were performed. The resolution of flavanols was adequate at a flow-rate of $1.2 \mathrm{ml} / \mathrm{min}$; however, it was not sufficient for dihydrochalcones and flavonols. As a consequence, the optimized gradient includes a flow-rate program, decreasing the flow to $0.8 \mathrm{ml} / \mathrm{min}$ after $25 \mathrm{~min}$, as described in Table 1. In Fig. 1 a chromatogram of a neutral extract of apple must is presented. As can be seen, the resolution between $\mathrm{B} 1, \mathrm{~B} 2, \mathrm{~B} 5$, an unknown trimer, trimer $\mathrm{Cl}$ and a procyanidin tetramer is good (e.g., $R_{\mathrm{s}}>1.25$ for trimer $\mathrm{Cl}$ and tetramer), being higher than that reported by Suárez et al. [4] $\left(R_{\mathrm{s}}<0.8\right.$ for trimer $\mathrm{Cl}$ and a procyanidin tetramer), who employed a $\mathrm{C}_{18}$ reversed-phase. What is more, the analytical method



Fig. 1. Separation of neutral polyphenolic compounds in apple must. Peaks: $1=(+)$-catechin; $2=$ cinnamic ester $\left(\lambda_{\max }=312.0\right.$ $\mathrm{nm}) ; 3=$ procyanidin $\mathrm{B} 1 ; 4=(-)$-epicatechin; $5=$ chlorogenic acid; $6=$ procyanidin B2: $7=$ unknown trimer; $8=$ trimer $\mathrm{Cl} ; 9=$ tetramer D; $10=$ procyanidin B5; $11=$ phloretin xyloglucoside; $12=$ phloridzin; $13=$ rutin + isoquercitrin; $14=$ unknown flavonol; $15=$ cinnamic ester $\left(\lambda_{\max }=312.0 \mathrm{~nm}\right): 16=$ avicularin + unknown flavonol; $17=$ quercitrin; $18=$ cinnamic ester $\left(\lambda_{\text {rax }}=326.3 \mathrm{~nm}\right)$. Chromatographic conditions as given in Table 1 and Section 2.

developed provided a good separation of other flavonoids and it is less time-consuming than those described elsewhere [3-5].

\subsection{Gradient optimization for phenolic acids in musts}

The main phenolic acids found in the apple musts studied, chlorogenic acid and a derivative of $p$ coumaric acid, with an absorbance maximum at 312 $\mathrm{nm}$, probably $p$-coumarylquinic, were well separated on the same column using chromatographic conditions similar to neutral phenolics (see Section 2). In Fig. 2 a chromatogram of an acidic phenolic extract of an apple must is shown. 


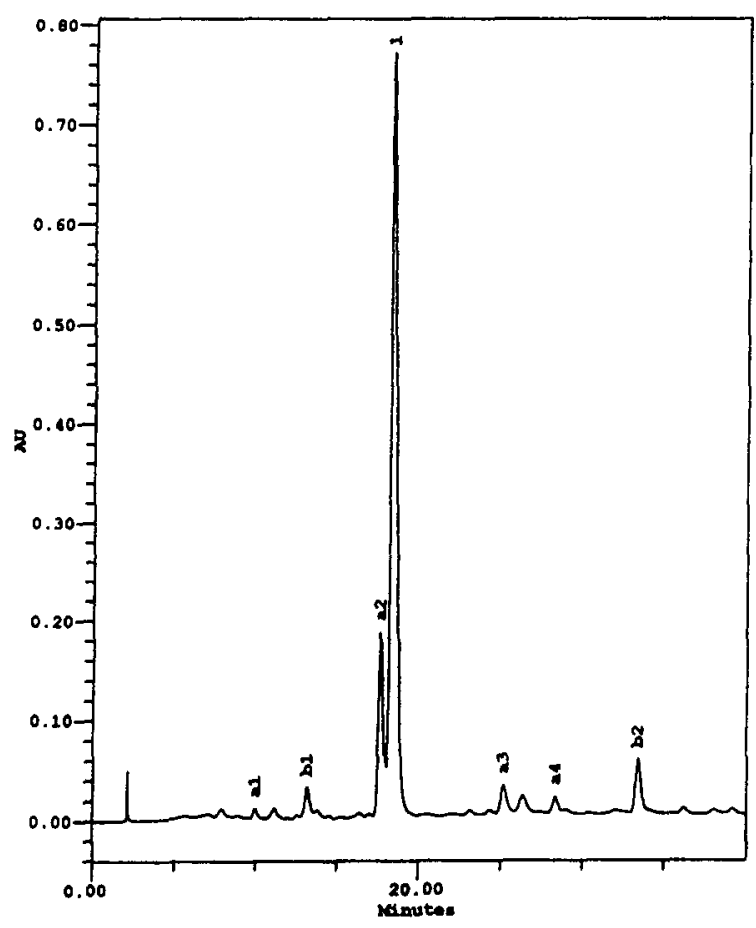

Fig. 2. Acidic phenolic extract of an apple must. Peaks: $1=$ chlorogenic acid; a1,a2,a3 $=p$-coumaric derivatives $\left(\lambda_{\max }=312.0\right.$ $\mathrm{nm}) ; \mathrm{b} 1, \mathrm{~b} 2=$ hydroxycinnamic acid derivatives $\left(\lambda_{\max }=326.3 \mathrm{~nm}\right.$, shoulder at $297.7 \mathrm{~nm}$ ). Chromatographic conditions as in Fig. I.

\subsection{Gradient optimization for phenolic acids in ciders}

Attempts to separate the different acidic phenols in ciders using the Hexyl reversed-phase cartridge were unsuccessful. At the initial conditions tested $(\mathrm{pH} 2.8$;

Table 2

Effect of the water washing volume on the percentage of phenolic compounds in the neutral extract of apple must

\begin{tabular}{lcrrr}
\hline $\begin{array}{l}\text { Solid-phase } \\
\text { supports }\end{array}$ & $\begin{array}{l}\text { Washing water } \\
(\mathrm{ml})\end{array}$ & CAT & EPI & CA \\
\hline Waters (360) & 2 & 100.0 & 100.0 & 76.7 \\
& 3 & 94.9 & 87.8 & 50.4 \\
& 4 & 70.2 & 91.3 & 44.0 \\
Lida (300) & 2 & 100.0 & 100.0 & 45.5 \\
& 3 & 100.0 & 100.0 & 14.7 \\
& 8 & 49.0 & 75.0 & 8.0 \\
Lida (1000) & 5 & 100.0 & 100.0 & 9.0 \\
& 10 & 90.0 & 96.2 & 4.2 \\
\hline
\end{tabular}

$\mathrm{CAT}=(+)$-catechin; EPI $=(-)$-epicatechin; $\mathrm{CA}=$ chlorogenic acid. $30^{\circ} \mathrm{C}$; ionic strength $0.01 \mathrm{M}$ ), overlapping of $p$ hydroxybenzoic and hydrocaffeic acid, and coelution of caffeic, vanillic and hydrocoumaric acid, and syringic and $p$-coumaric acids were observed. Variations in the mobile-phase composition such as $\mathrm{pH}$ (2.6-4.5), ionic strength $(0-0.1 M)$, temperature $\left(25-45^{\circ} \mathrm{C}\right)$, or elution profiles did not improve separation. Fig. 3 compares two chromatograms of a synthetic phenolic acid mixture, using a Nucleosil $\mathrm{C}_{18}$ (Fig. 3A) and a $\mathrm{C}_{6}$ (Fig. 3B) column, showing that the resolution for phenolic acids of the $\mathrm{C}_{18}$ column is better than that of the $\mathrm{C}_{6}$ one, which may be due to the smaller particle diameter of the $C_{18}$ phase, and a higher retention possibly caused by the lower polarity of this phase. Resolutions $\left(R_{\mathrm{s}}\right)$ were greater than 1.0 for all phenolic acids on the $C_{18}$ reversed-phase column (Fig. 3A); whereas $R_{\mathrm{s}}<0.6$ for vanillic, caffeic, hydrocoumaric, syringic and $p$-coumaric acids, and $R_{\mathrm{s}}=0.8$ for $p$-hydroxybenzoic and hydrocaffeic acids were obtained on the $C_{6}$ column (Fig. 3B).

Major acidic polyphenols in cider are $p$-coumaric and caffeic acid derivatives, as well as their reduced forms, such as hydrocoumaric and hydrocaffeic acids (Fig. 4), which occur in cider due to metabolism of hydroxycinnamic derivatives by species of $\mathrm{Lac}$ tobacillus [17].

\subsection{Optimization of the extraction conditions}

$\mathrm{C}_{18}$ solid-phase supports with different physical characteristics (carbon load, pore and particle size) were tested, searching for the optimal fractionation ability (less than $10 \%$ of phenolic acids in the neutral extract, with minimum losses of neutral compounds).

Conditioning and loading of solid-phase supports were performed as described in Section 2, using an apple must for optimization studies. After neutral phenolics were loaded, an additional washing step with water was necessary in order to eliminate phenolic acids remaining as interferents in the neutral extract. This step was critical, as reported in Table 2. Cartridges from Waters had low fractionation ability, as shown by the high percentage of chlorogenic acid remaining in the neutral extract. Increase of the water washing volume gave losses of catechin, although epicatechin was less affected. In contrast, Lida (300 mg) solid-phase supports had a 
Table 3

Recovery study of some phenolic compounds in apple must and cider

\begin{tabular}{lcccl}
\hline & $\begin{array}{l}\text { Amount } \\
(\mu \mathrm{g} / \mathrm{ml} ; \mathrm{avg} \pm \text { R.S.D. })\end{array}$ & $\begin{array}{l}\text { Amount added } \\
(\mu \mathrm{g} / \mathrm{ml})\end{array}$ & $\begin{array}{l}\text { Recovery } \\
(\%)\end{array}$ & $\begin{array}{l}\text { Liquid-liquid extraction }{ }^{4} \\
(\% \pm \text { R.S.D. })\end{array}$ \\
\hline Juice & & & & \\
Chlorogenic acid & $186.9 \pm 4.2$ & 152.8 & $100 \pm 6.1$ & $68 \pm 25.0$ \\
Epicatechin & $18.4 \pm 0.6$ & 33.5 & $102 \pm 1.1$ & $79 \pm 15.2$ \\
Phloridzin & $21.2 \pm 1.1$ & 42.5 & $111 \pm 2.4$ & - \\
& & 104.3 & $106 \pm 0.3$ & - \\
Quercitrin & $5.1 \pm 0.3$ & 10.2 & $103 \pm 4.2$ & - \\
Cider & & & & \\
Hydrocaffeic acid & $47.6 \pm 4.0$ & 58.0 & $84 \pm 4.6$ & - \\
Caffeic acid & $20.6 \pm 4.5$ & 29.8 & $91 \pm 3.0$ & $75 \pm 6.2$ \\
$p$-Coumaric acid & $52.2 \pm 4.9$ & 51.2 & $107 \pm 4.2$ & $82 \pm 5.1$ \\
Epicatechin & - & 60.0 & $95 \pm 5.9$ & \\
Phloridzin & $64.5 \pm 1.6$ & 68.0 & $105 \pm 1.1$ & \\
\hline
\end{tabular}

"See Ref. [21].
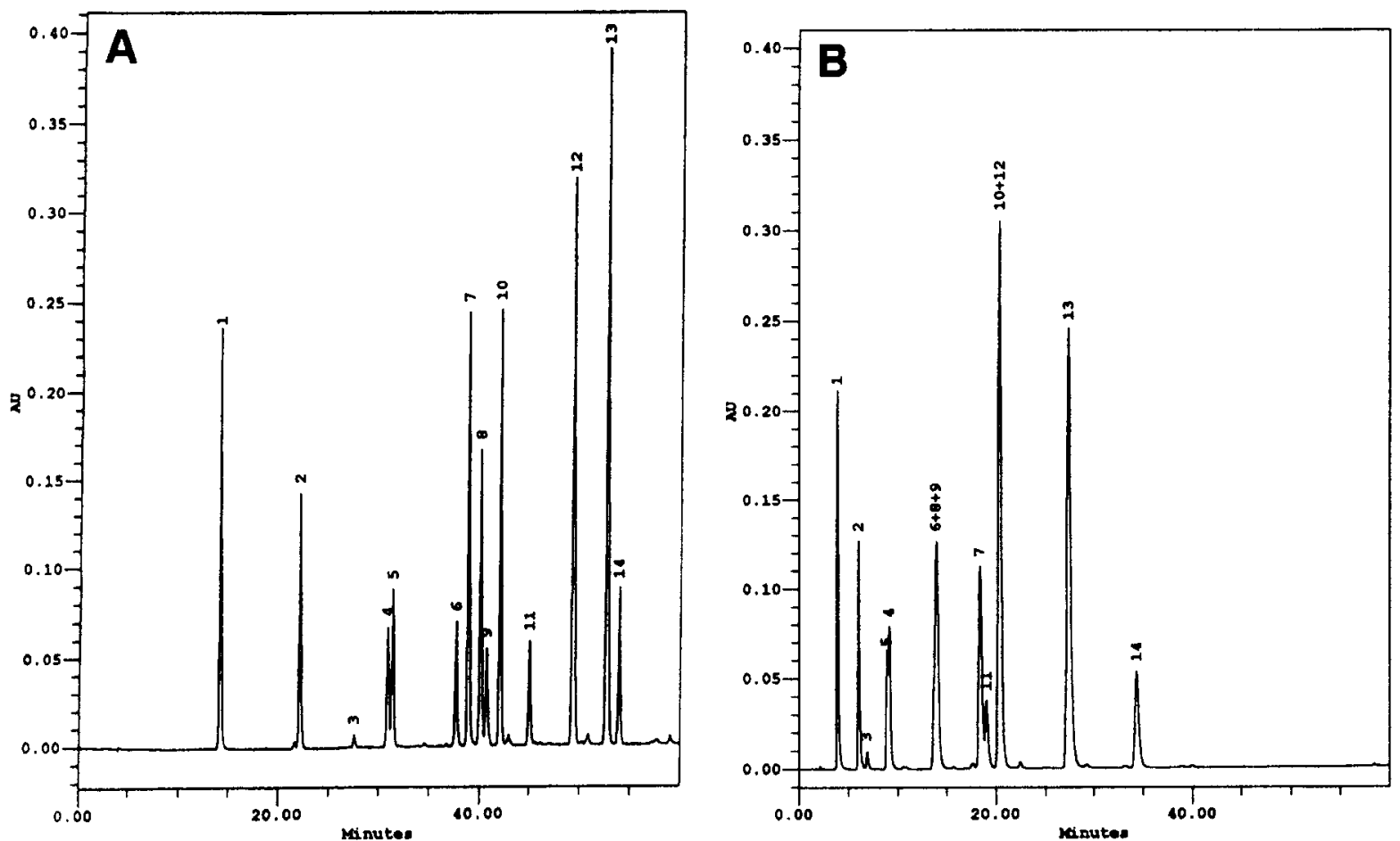

Fig. 3. Chromatogram of a synthetic phenolic acid mixture. Peaks: $1=$ gallic acid; $2=$ protocatechuic acid; $3=$ gentisic acid; $4=p$ hydroxybenzoic acid; $5=$ hydrocaffeic acid; $6=$ vanillic acid; $7=$ chlorogenic acid; $8=$ caffeic acid; $9=$ hydrocoumaric acid; $10=$ syringic acid; $11=$ hydroferulic acid; $12=p$-coumaric acid; $13=$ ferulic acid; $14=$ sinapic acid. (A) Chromatographic conditions: Nucleosil $120 \mathrm{C}_{16}$ ( $250 \times 4.6 \mathrm{~mm} \mathrm{I.D.,} 3 \mu \mathrm{m}$ particle size), $2 \%$ acetic acid (solvent A) and methanol (solvent B); gradient elution as given in Section 2. (B) Chromatographic conditions: Spherisorb $C_{6}(250 \times 4.6 \mathrm{~mm}$. I.D., $5 \mu \mathrm{m}$ particle size), $2 \mathrm{mM}$ phosphoric acid (solvent $A)$ and methanol (solvent $\mathrm{B}$ ); gradient elution as shown in Table 1 . 


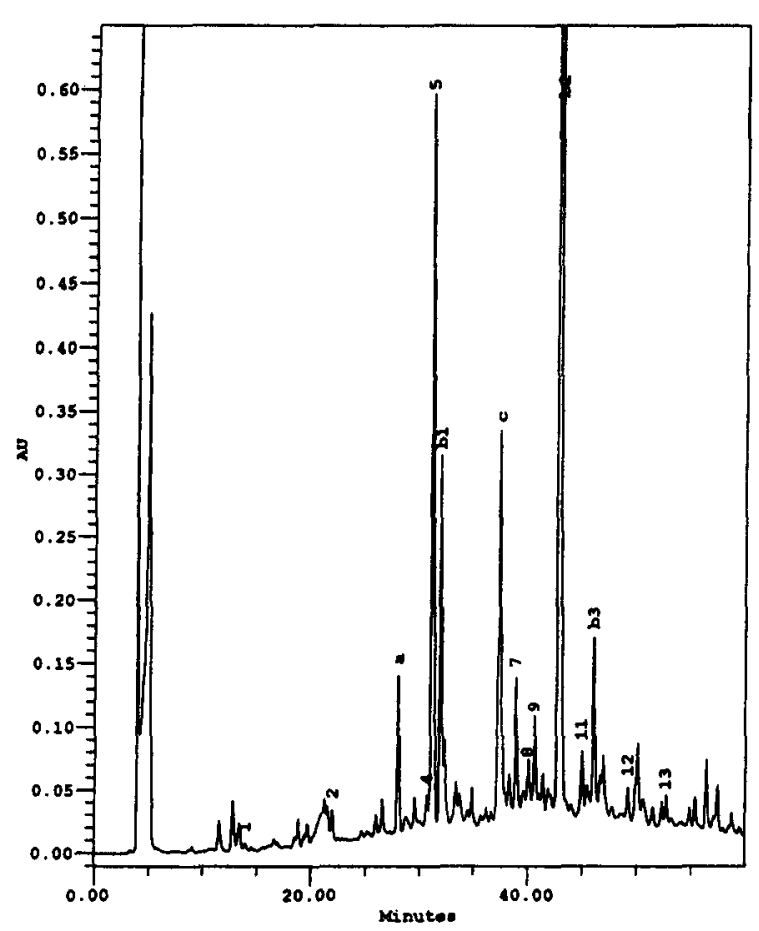

Fig. 4. Chromatogram of an acidic phenolic extract of cider. Peaks and chromatographic conditions as in Fig. 3A. Unknown peaks: $\mathrm{a}=$ hydrocoumaric acid type spectra $\left(\lambda_{\max }=274.0\right) ; \mathrm{b} 1, \mathrm{~b} 2, \mathrm{~b} 3=p$ coumaric acid type spectra $\left(\lambda_{\max }=312.0\right) ; \mathrm{c}=$ hydroxycinnamic acid type spectra $\left(\lambda_{\max }=326 \mathrm{~nm}\right.$, shoulder at $\left.297.7 \mathrm{~nm}\right)$.

lower percentage of chlorogenic acid in the neutral portion. Thus, $1-\mathrm{g} \mathrm{C}_{18}$ supports from Lida were tested in order to increase the sensitivity of the extraction process. The percentage of chlorogenic acid in the neutral extract decreased as the volume of water increased, with minimum flavanol losses (Table 2). Therefore, $10 \mathrm{ml}$ of $\mathrm{pH} 7.0$ water was chosen for apple musts. The extraction procedure required an additional washing step of the acidic cartridge with $5 \mathrm{ml}$ of $0.01 \mathrm{M} \mathrm{HCl}$ to eliminate some residual sugars, which interfere in the evaporation of the acidic eluate. This step should be avoided in ciders, since these samples do not contain sugars, and losses of some phenolic acids were observed.

The different retention behavior could be explained by the physical characteristics of the packing materials. Cartridges from Waters showed higher carbon loading and pore size $(12 \% ; 125 \AA)$ than Lida cartridges $(5.91-6.54 \% ; 60 \AA)$. It has been reported for materials with pore sizes between 60 and $100 \AA$, that a higher average pore size increases the retention capacity, due to stronger interactions between the nonpolar surface and the analyte [18]. Also, a close linear relationship between carbon loading and capacity factor was found [18]. The higher carbon loading and pore diameter of cartridges from Waters might account for the greater retention of the ionized form of chlorogenic acid (Table 2).

\subsection{Recovery studies}

Recovery studies were done in duplicate. Known amounts of polyphenols were added to apple juice and cider samples, and the resulting spiked samples were subjected to the entire analytical sequence (Table 3). Recoveries ranged between $100 \%$ and $110 \%$ for all compounds tested in apple juice, and between $84 \%$ and $107 \%$ for cider. Repeatability between two extractions was high (R.S.D. less than $5 \%$ ).

The recoveries determined for different kinds of phenolics in apple products (apple must and cider) by means of the proposed solid-phase extraction (SPE) system were adequate for analytical purposes, and in accordance with the results obtained by Jaworski and Lee [10] and Oszmianski and Lee [19] in grape, and Kermasha et al. [20] in maple products.

The present analytical method is simple and easy to use, and it is more effective for the quantitation of major phenolic compounds in apple musts and ciders, compared to the liquid-liquid extraction method [21].

\section{Acknowledgments}

The authors are indebted to the Comision Interministerial de Ciencia y Tecnología (CICYT ALI921027-C03-02) and the Principado de Asturias for financial support.

\section{References}

[1] A.G.H. Lea and G.M. Arnold, J. Sci. Food Agric., 29 (1978) 478.

[2] M.H. Salagoity-Auguste and A. Bertrand, J. Sci. Food Agric., 35 (1984) 1241. 
[3] E. Delage, G. Bohuon, A. Baron and J.F. Drilleau, J. Chromatogr., 555 (1991) 125.

[4] B. Suárez, J. Santamaría, J.J. Mangas and D. Blanco, J. Agric. Food Chem., 42 (1994) 2732.

[5] F.J. Pérez-Ilzarbe, V. Martínez, T. Hernández and I. Estrella, J. Liq. Chromatogr., 15 (1992) 637.

[6] G.A. Spanos, R.E. Wrolstad and D.A. Heatherbell, J. Agric. Food Chem., 38 (1990) 1572.

[7] A.G.H. Lea and C.F. Timberlake, J. Sci. Food Agric.. 25 (1974) 1537.

[8] G.A. Spanos and R.E. Wrolstad, J. Agric. Food Chem., 38 (1990) 1565.

[9] R.L. Rouseff, G.R. Dettweiler and R.M. Swaine, J. Chromatogr. Sci., 30 (1992) 383.

10] A.W. Jaworki and C.Y. Lee, J. Agric. Food Chem., 35 (1987) 257.

111 S. Burda, W. Oleszek and C.Y. Lee, J. Agric. Food Chem., 38 (1990) 945 .
[12] M.Y. Coseteng and C.Y. Lee, J. Food Sci., 52 (1987) 985.

[13] J. Oszmianski, T. Ramos and M. Bourzeix, Am. J. Enol. Vitic., 39 (1988) 259.

[14] A.G.H. Lea, J. Chromatogr., 238 (1982) 253.

[15] F. Montreau, Conn. Vigne Vin., 6 (1972) 397.

[16] A.G.H. Lea, J. Chromatogr., 194 (1980) 62.

[17] F.W. Beech and J.G. Carr, in A.H. Rose (Editor), Alcoholic Beverages, Academic Press, London, 1977, Ch. 3, p. 188.

[18] C. Horváth. in C.F. Simpson (Editor), Techniques in Liquid Chromatography, John Wiley \& Sons, New York, 1982, Ch. 10, p. 264.

[19] J. Oszmianski and C.Y. Lee, Am. J. Enol. Vitic., 3 (1990) 204.

[20] S. Kermasha, M. Goetghebeur and J. Dumont. J. Agric. Food Chem., 43 (1995) 708.

[21] B. Fernández de Simón, J. Pérez-Ilzarbe, T. Hernández, C. Gómez-Cordovés and I. Estrella, Chromatographia, 30 (1990) 35 